\title{
A cerclagem para prevenção da prematuridade: para quem indicar?
}

\author{
Cerclage for the prevention of prematurity: for whom should it be indicated?
}

\author{
Rosiane Mattar ${ }^{1}$
}

Palmer e Lacomme, em 1948, na França, e Lash e Lash, em 1950, nos Estados Unidos da América divulgaram ao conhecimento médico condição que ficou conhecida como insuficiência istmocervical (IIC), em que há perda gestacional recorrente na forma de abortos tardios e/ou partos prematuros iniciados por cervicodilatação precoce, provocada por defeito local e não pela presença de contrações uterinas.

As mulheres portadoras desta doença apresentam história característica, em que a dilatação do colo uterino se dá sem sintomas até que haja a rotura das membranas devida à exposição das mesmas ao ambiente vaginal, o que é seguido por trabalho de parto ou de abortamento rápido, pouco doloroso e sem sangramento expressivo. A criança nasce viva, mas sofre índices elevados de morbimortalidade em razão da prematuridade. Para evitar este desfecho ocasionado pela IIC, logo depois da descrição da doença, a cerclagem foi sugerida como tratamento capaz de evitar esta perda gestacional.

A primeira técnica foi a proposta por Shirodkar em 1953, e previa que a colocação da sutura fosse realizada após a abertura da mucosa vaginal. Em 1957, foi sugerida por McDonald a técnica de cerclagem por via transmucosa, mais simples e com menos complicações. Estas duas técnicas são realizadas por via vaginal e constituem a base de todas as variações descritas até o momento. Benson e Durfee, em 1965, descreveram a cerclagem realizada por via abdominal para aqueles casos em que a via vaginal fosse impossibilitada pela ausência ou irregularidade acentuada do colo uterino.

Cerclagem significa sutura em bolsa e foi idealizada como maneira de manter o colo fechado, impossibilitando anatomicamente sua dilatação antes do final da gravidez, evitando, assim, a prematuridade. Inicialmente indicada nas pacientes com perdas gestacionais com história de IIC e nos casos de cervicodilatação com exposição das membranas, como tentativa heróica de salvar aquela gravidez, ela parecia garantir bons resultados.

Pela facilidade de sua realização e pelos bons resultados obtidos nos casos de IIC, como classicamente ela foi definida, esta cirurgia começou a ter suas indicações cada vez mais ampliadas para outras ocasiões em que a prematuridade era temida.

Foi tentada em casos de placenta de inserção baixa, com a perspectiva de que a sutura determinaria que o colo ficasse fechado e a área do orificio interno não se alteraria, e assim garantiria menos risco de sangramento e prematuridade. Esta indicação no entanto, foi abandonada, após relativamente poucos casos clínicos, por ter sido observado que não impedia os episódios de hemorragia e não melhorava o prognóstico materno-fetal.

Uma outra indicação da cerclagem surgiu com o conceito do colo uterino curto durante a gravidez, conceito este que já existia pela observação clínica através do toque vaginal, mas que se ampliou com o uso da ultra-sonografia.

A introdução e a ampliação da utilização da ultra-sonografia em Obstetrícia, particularmente dos transdutores transvaginais, permitiu avaliar com muita precisão as medidas e a forma do colo uterino durante a gestação e trouxe o conhecimento de que a cérvice uterina tem diferentes comprimentos em mulheres diversas, e que quanto menor o colo, maior é o risco de prematuridade.

Existem controvérsias sobre o limite de comprimento representativo de risco, 25, 20 ou 15 $\mathrm{mm}$, havendo tendência dos estudos em adotar o ponto de corte de $20 \mathrm{~mm}$, medido entre 22 e 24 semanas de gravidez. Além disto, outros marcadores de risco de prematuridade têm sido avaliados, como o volume do colo, o sinal de afunilamento do orificio interno e a ausência do eco glandular endocervical, sem que seu real valor tenha sido estabelecido ${ }^{1-5}$.

1 Professora Livre-Docente do Departamento de Obstetrícia da Universidade Federal de São Paulo - Escola Paulista de Medicina (UNIFESP-EPM) São Paulo (SP), Brasil.

Correspondência:

Rua Dr Diogo de Faria, 477 - apto 31 - 04037-000 - São Paulo - SP - Fone: (11) 55759458, (11) 96097979 - e-mail: rosiane.toco@epm.br 
O reconhecimento de que o colo curto se associa a nascimentos pré-termo e a analogia com a IIC clássica fizeram com que se indicasse a cerclagem como tentativa de evitar a prematuridade. Assim, assistimos nos últimos anos a enorme aumento da aplicação desta cirurgia em pacientes com colo denominado curto, com ou sem a vivência de partos prematuros prévios.

Nas últimas décadas também assistimos a maior procura pelos métodos de reprodução assistida e melhores resultados na obtenção de gestações, o que provocou aumento expressivo no número de gestações múltiplas, acompanhadas pelo risco da prematuridade inerente a elas. Também nestes casos as modificações do colo uterino, como esvaecimento e dilatação precoces, muitas vezes observados nas gestações múltiplas, fizeram com que presenciássemos a crescente indicação de cerclagem para estes casos, com o intuito de propiciar maior idade gestacional dos conceptos ao nascimento e melhorar a sobrevida dos mesmos.

Devemos ponderar, entretanto, quais os beneficios e riscos que acompanham este número crescente de cerclagens. Hoje, decorridos 50 anos de uso da cerclagem, a prematuridade continua a representar grave problema obstétrico, valoriza-se a prática da medicina baseada em evidências e a literatura permanece polarizada em relação à eficácia da cerclagem.

Os primeiros ensaios clínicos para avaliação da eficácia da cerclagem em pacientes com IIC foram realizados por Rush et $a l .{ }^{6}$ e Lazar et al. ${ }^{7}$, que referiram não ter encontrado diferença significativa no prognóstico das pacientes submetidas à cirurgia e comparadas àquelas para as quais se adotou o repouso. Pelo contrário, observaram nas primeiras maior tempo de hospitalização e febre. No entanto, estes estudos foram realizados em grupos populacionais pequenos, e não alcançaram repercussão no meio médico.

O primeiro grande impacto foi fornecido por estudo multicêntrico, coordenado pelo Royal College of Obstetricians and Gynaecologists ${ }^{8}$, que analisou 1292 mulheres e observou no grupo submetido à cirurgia maior tempo de hospitalização, maior uso de tocoliticos e índices de morbidade febril mais elevados, indicando que a cerclagem era importante para redução de prematuridade somente no grupo de alto risco, isto é, nas mulheres com prematuridade prévia. Foi demonstrado em metaanálise com estes estudos que somente um parto antes de 33 semanas de gestação é obtido com a prática de 25 cerclagens em pacientes com diagnóstico de IIC.

Em pacientes com risco prévio de prematuridade, Althuisius et al. ${ }^{9}$ verificaram menor freqüência de rotura prematura de membranas e parto pré-termo. Odibo et al. ${ }^{10}$, em meta-análise, observaram tendência para prevenção de parto pré-termo com menos que 34 semanas, pelo uso da cerclagem, nas pacientes de risco, isto é, as com perdas gestacionais de segundo trimestre anteriormente.

No caso da cerclagem de urgência, praticada na vigência de cervicodilatação e exposição das membranas, alguns relatos referem que com ela se alcança maior idade gestacional ao nascimento ${ }^{11}$, prolongando a gravidez, em média, 7 semanas $^{12}$, porém com índices elevados de corioamnionite. Por outro lado, em ensaio clínico, Althuisius et al. ${ }^{13}$ referiram que, embora ela determine maior idade gestacional ao nascimento, observa-se o mesmo índice de sobrevida neonatal.

Não existem estudos randomizados adequados e não há conduta estabelecida para estes casos. Entretanto, há que se ter muito bom senso na indicação da cirurgia nestas condições, principalmente pelo risco de infecção intra-uterina, que pode provocar a necessidade de histerectomia posterior e a morte materna.

Quanto à cerclagem por via transabdominal, existe concordância em que deva ser indicada somente nos casos em que há impossibilidade da cirurgia por via vaginal, pois ela apresenta maiores riscos de morbidade materna, relacionada a casos de hemorragia na passagem da sutura, rotura das membranas, riscos da laparotomia e da cesárea ${ }^{14}$. Os últimos estudos têm mostrado que, nos casos de história clássica de IIC em que se indicou a cerclagem transabdominal, houve melhoria do prognóstico, com menos casos de parturição antes da $32^{\mathrm{a}}$ semana e bons índices de sobrevida fetal, que chegaram a atingir a cifra de $93,5 \%^{15}$. Há que se ressaltar, entretanto, que existem poucos casos descritos até o momento, não permitindo conclusão a respeito da eficácia e segurança desta cirurgia.

Em relação às pacientes com diagnóstico provável de IIC em que a gravidez anterior foi tratada com cerclagem e houve sucesso, há dúvida sobre a melhor conduta. Não existem estudos randomizados sobre estes casos e a maioria das mulheres tem medo de evoluir a gravidez futura sem a cerclagem, portanto tem sido recomendado que a decisão seja conjunta entre o médico e a paciente, e baseada no bom senso. 
Em gestantes com colo curto, embora os primeiros estudos tenham sugerido tratamento pela cerclagem, as últimas revisões têm questionado sua validade. Em colos com comprimento menor que $15 \mathrm{~mm}$, observou-se no grupo submetido a cerclagem a mesma incidência de prematuridade e maior freqüência de rotura prematura de membranas, sem diferença na sobrevida dos conceptos ${ }^{16}$ em comparação ao grupo não submetido ao procedimento. Em pacientes com colo menor que 25 $\mathrm{mm}$, Rust et $a{ }^{17}$ referiram que a cerclagem não melhorou os índices de prematuridade e suas complicações, enquanto Althuisius et al. ${ }^{18}$ observaram que ela aumentou a idade gestacional do parto, mas não aumentou a sobrevida neonatal. Ao se estudarem as diferentes idades gestacionais do parto, não foi detectada diferença na ocorrência de parto prematuro antes da $34^{\mathrm{a}}, 32^{\mathrm{a}}$ ou $28^{\mathrm{a}}$ semana ou nos índices de mortalidade ou morbidade neonatal em pacientes com colo curto submetidas à cerclagem ${ }^{19,20}$.

No maior estudo descrito até o momento, To et al. ${ }^{21}$, analisando 47.123 mulheres de baixo risco observaram que 470 apresentavam colo com comprimento menor que $15 \mathrm{~mm}$. Entre as 253 submetidas a estudo randomizado, não houve diferença na proporção de partos antes da $33^{a}$ semana, na mortalidade e morbidade perinatal ou materna entre os grupos com cerclagem e o de conduta expectante.

Em pacientes com gestação múltipla, em que foi indicada a cerclagem profilática, não foi verificado menor índice de parto prematuro ou melhoria na idade gestacional ${ }^{22-24}$. Já se tentou basear a indicação da cerclagem nas gestações múltiplas à identificação de colo curto ao US, mas também nestes casos não se verificou melhor prognóstico em relação à ocorrência de parto prematuro $^{25}$.

Observamos com preocupação a crescente onda de indicação de cerclagem. Esta cirurgia tornou-se tão integrada na prática obstétrica que os obstetras têm dificuldade em não utilizá-la ${ }^{12}$, mas já está definido que não há vantagens em se indicar cerclagem para pacientes de baixo ou médio risco de perda gestacional de segundo trimestre ${ }^{26}$.

Ela não deve ser indicada para o tratamento do colo curto rastreado pelo US, principalmente nas mulheres sem fator de risco para prematuridade ${ }^{27}$. Também não há que se praticar a cerclagem em pacientes com gestação múltipla.

A cerclagem permanece como opção terapêutica somente para as pacientes de alto risco, com história clássica de IIC e nos casos de cervicodilatação em que se indica a cirurgia de urgência, desde que não haja contra-indicações.

Julgamos que o bom senso deve permear as indicações cirúrgicas, sempre tendo em mente que não é verdade que a cerclagem seja cirurgia simples e de poucos riscos, e que na dúvida sobre sua necessidade ou não, seria melhor realizá-la. Devemos disseminar no Brasil o conhecimento de que diversas complicações podem advir desta intervenção, inclusive a perda da capacidade reprodutora e a vida materna, e o fato de que, ao acreditar que a realização da cerclagem está auxiliando aquela gestação, podemos estar deixando de identificar e tratar a verdadeira causa da prematuridade.

\section{Referências}

1. Iams JD, Goldenberg RL, Meis PJ, Mercer BM, Moawad A, Das A, et al. The length of the cervix and the risk of spontaneous premature delivery. National Institute of Child Health and Human Development Maternal Fetal Medicine Unit Network. N Engl J Med. 1996;334(9):567-72.

2. Oliveira TA, Carvalho CMP, Souza E, Santos JFK, Guaré SO, Mariani Neto C, et al. Avaliação do risco de parto prematuro: teste de fibronectina fetal e medida do colo uterino. Rev Bras Ginecol Obstet. 2002;22(10):633-9.

3. Carvalho MHB, Bittar RE, Gonzáles M, Brizot ML, Zugaib M. Avaliação do risco para parto prematuro espontâneo pelo comprimento do colo uterino no primeiro e segundo trimestres da gravidez. Rev Bras Ginecol Obstet. 2002;24(7):463-8.

4. Freitas-Júnior RAO, Mauad-Filho F, Duarte G, Ferreira AC, Freitas AKMSO, Azevedo GD. Evolução do comprimento cervical uterino na gestação, avaliado pela ultra-sonografia transvaginal. Rev Bras Ginecol Obstet. 2003;25(2):115-21.

5. Pires CR, Moron AF, Mattar R, Kulay Júnior L. Avaliação do comprimento do colo e da ausência do eco glandular endocervical para predição do parto pré-termo. Rev Bras Ginecol Obstet. 2004;26(3):193-200. 
6. Rush RW, Isaacs S, McPherson K, Jones L, Chalmers I, Grant A. A randomized controlled trial of cervical cerclage in women at high risk of spontaneous preterm delivery. Br J Obstet Gynaecol. 1984;91(8):724-30.

7. Lazar P, Gueguen S, Dreyfus J, Renaud R, Pontonnier G, Papiernik E. Multicentred controlled trial of cervical cerclage in women at moderate risk of preterm delivery. Br J Obstet Gynaecol. 1984;91(8):731-5.

8. Final report of the Medical Research Council/Royal College of Obstetricians and Gynaecologists multicentre randomised trial of cervical cerclage. MRC/RCOG Working Party on Cervical Cerclage. Br J Obstet Gynaecol. 1993;100(6):516-23.

9. Althuisius SM, Dekker GA, van Geijn HP, Bekedam DJ, Hummel P. Cervical incompetence prevention randomized cerclage trial (CIPRACT). Am J Obstet Gynecol. 2000;183(4):823-9.

10. Odibo AO, Farrell C, Macones GA, Berghella V. Development of a scoring system for predicting the risk of preterm birth in women receiving cervical cerclage. J Perinatol. 2003;23(8):664-7.

11. Mattar R, Matheus ED, Mendes ETR, Stefano T, Kobayashi S, Santana RM, et al. O tratamento da insuficiência istmocervical com protrusão de membranas. Rev Bras Ginecol Obstet. 1999;21(3):171-4.

12. Owen J, Iams JD, Hauth JC. Vaginal sonography and cervical incompetence. Am J Obstet Gynecol. 2003; 188(2):586-96.

13. Althuisius SM, Dekker GA, Hummel P, van Geijn HP. Cervical incompetence prevention randomized cerclage trial: emergency cerclage with bed rest versus bed rest alone. Am J Obstet Gynecol. 2003;189(4):90710.

14. Turnquest MA, Britton KA, Brown HL. Outcome of patients undergoing transabdominal cerclage: a descriptive study. J Matern Fetal Med. 1999;8(5):225-7.

15. Lotgering FK, Gaugler-Senden IP, Lotgering SF, Wallenburg HC. Outcome after transabdominal cervicoisthmic cerclage. Obstet Gynecol. 2006;107(4):779-84.

16. Hassan SS, Romero R, Maymon E, Berry SM, Blackwell SC, Treadwell MC, et al. Does cervical cerclage prevent preterm delivery in patients with a short cervix? Am J Obstet Gynecol. 2001;184(7):1325-9.

17. Rust OA, Atlas RO, Jones KJ, Benham BN, Balducci J. A randomized trial of cerclage versus no cerclage among patients with ultrasonographically detected second-trimester preterm dilatation of the internal os. Am J Obstet Gynecol. 2000;183(4):830-5.

18. Althuisius SM, Dekker GA, Hummel P, Bekedam DJ, van Geijn HP. Final results of the Cervical Incompetence Prevention Randomized Cerclage Trial (CIPRACT): therapeutic cerclage with bed rest versus bed rest alone. Am J Obstet Gynecol. 2001;185(5):1106-12.

19. Belej-Rak T, Okun N, Windrim R, Ross S, Hannah ME. Effectiveness of cervical cerclage for a sonographically shortened cervix: a systematic review and meta-analysis. Am J Obstet Gynecol. 2003;189(6):1679-87.

20. Berghella V, Odibo AO, To MS, Rust OA, Althuisius SM. Cerclage for short cervix on ultrasonography: meta-analysis of trials using individual patient-level data. Obstet Gynecol. 2005;106(1):181-9.

21. To MS, Alfirevic Z, Heath VC, Cicero S, Cacho AM, Williamson PR, et al. Cervical cerclage for prevention of preterm delivery in women with short cervix: randomised controlled trial. Lancet. 2004;363(9424):184953.

22. Newman RB, Krombach RS, Myers MC, McGee DL. Effect of cerclage on obstetrical outcome in twin gestations with a shortened cervical length. Am J Obstet Gynecol. 2002;186(4):634-40.

23. Parilla BV, Haney EI, MacGregor SN. The prevalence and timing of cervical cerclage placement in multiple gestations. Int J Gynaecol Obstet. 2003;80(2):123-7.

24. Rebarber A, Roman AS, Istwan N, Rhea D, Stanziano G. Prophylactic cerclage in the management of triplet pregnancies. Am J Obstet Gynecol. 2005;193(3 Pt 2):1193-6.

25. Roman AS, Rebarber A, Pereira L, Sfakianaki AK, Mulholland J, Berghella V. The efficacy of sonographically indicated cerclage in multiple gestations. J Ultrasound Med. 2005;24(6):763-8.

26. Drakeley AJ, Roberts D, Alfirevic Z. Cervical stitch (cerclage) for preventing pregnancy loss in women. Cochrane Database Syst Rev. 2003;(1):CD003253.

27. American College of Obstericians and Gynecologists. ACOG Practice Bulletin. Cervical insufficiency. Obstet Gynecol. 2003;102(5 Pt 1):1091-9 